\title{
Immunomodulatory properties of graphene oxide for osteogenesis and angiogenesis
}

This article was published in the following Dove Press journal:

International Journal of Nanomedicine

\author{
Deting Xue ${ }^{1, *}$ \\ Erman Chen ${ }^{1, *}$ \\ Huiming Zhong ${ }^{2, *}$ \\ Wei Zhang' \\ Shengdong Wang' \\ Muhammad Umar Joomun' \\ Tianyi Yao ${ }^{3}$ \\ Yanbin Tan' \\ ShiSheng $\operatorname{Lin}^{3}$ \\ Qiang Zheng' \\ Zhijun Pan' \\ 'Department of Orthopaedics, The \\ Second Affiliated Hospital, Zhejiang \\ University School of Medicine, \\ Zhejiang University, Hangzhou \\ 310009, People's Republic of China; \\ ${ }^{2}$ Department of Emergency, The \\ Second Affiliated Hospital, Zhejiang \\ University School of Medicine, \\ Zhejiang University, Hangzhou \\ 310009, People's Republic of China; \\ ${ }^{3}$ Department of Information Science \\ and Electronic Engineering and State \\ Key Laboratory of Modern Optical \\ Instrumentation, Zhejiang University, \\ Hangzhou 3 I 0027, People's Republic \\ of China
}

*These authors contributed equally to this work

Correspondence: Deting Xue; Zhijun Pan Department of Orthopaedics, The Second Affiliated Hospital, Zhejiang University School of Medicine, Zhejiang University, 88 jiefang Road, Hangzhou 310009 , People's Republic of China

Tel +86 57I 8778 3530;

$+8657 \mid 87783533$

Fax +86 57I 87022776

Email blueskine@zju.edu.cn;

zepzj@zju.edu.cn

\begin{abstract}
Background: The osteo-immunomodulatory properties of biomaterials play an important role in the outcomes of bone regeneration. Graphene oxide (GO) has been widely applied in many research fields due to its unique properties. However, the immunomodulatory properties of GO as a biomaterial for bone tissue engineering are still unclear.
\end{abstract}

Materials and methods: In this study, we evaluated the Inflammatory response of RAW264.7 cells influenced by GO. Then the osteogenic differentiation of BMSCs, and angiogenic differentiation of human umbilical vein endothelial cells (HUVECs) by stimulation with GO/RAW 264.7-conditioned culture medium were accessed. We also further investigated the possible mechanisms underlying the osteo- and angio-immunomodulatory effects of GO.

Results: Our results showed that GO stimulates the secretion of oncostatin M, tumor necrosis factor alpha and other factors through the nuclear factor- $\kappa B$ pathway. GO/RAW264.7-conditioned medium promoted the osteogenic differentiation of BMSCs, stimulated upregulation of the HUVECs of vascular-related receptors, and promoted their tube formation in vitro.

Conclusion: In conclusion, our research shows that GO, as a biomaterial, can induce the formation of a beneficial osteo-immunomodulatory environment and is a promising biomaterial for bone tissue engineering.

Keywords: graphene oxide, osteo-immunomodulatory, bone marrow mesenchymal stem cells, osteogenesis, angiogenesis, RAW264.7 cells, HUVECs

\section{Introduction}

In recent years, tissue engineering, which involves seeding cells and a three-dimensional (3D) biomaterial scaffold, has become a promising approach for treating bone defects. ${ }^{1,2}$ With developments in materials science, biomaterial scaffolds are becoming safer and more multifunctional. Numerous studies have addressed how to improve the biocompatibility of biomaterials for seeding cell growth. ${ }^{3}$ However, biomaterial implants initiate interactions with the host tissue after implantation; this may exert a profound impact on the host immune response, which may in turn affect the healing and repair process. $^{4,5}$ As such, the osteo-immunomodulatory properties of biomaterials play an important role in the outcomes of bone regeneration. ${ }^{6,7}$ The traditional design of bone biomaterials mainly focuses on accelerating the osteogenic differentiation of the seeding cells, but fails to take into account the osteo-immunomodulatory properties of the biomaterial. To date, only limited studies have focused on this issue.

Several types of host cells collaboratively and sequentially participate in immune responses against the scaffolds. ${ }^{8}$ Macrophages (including their precursors, monocytes) are the first line of defense against foreign biomaterials. The inflammatory cytokines secreted by macrophages have been traditionally considered as more detrimental than beneficial with respect to the settlement of biomaterials in the body. 
However, increasing evidence has shown that an appropriate immune response is necessary for mediating tissue-scaffold integration. ${ }^{9,10}$ Some studies have shown that the inflammatory cytokines interleukin (IL)-4, IL-10, and IL13 have an osteogenic effect on bone formation, while tumor necrosis factor alpha (TNF- $\alpha)$, IL-6, and IL-1 have the opposite effect. ${ }^{11}$ Furthermore, immune cells could enhance neovascularization by secreting pro-angiogenic factors, such as vascular endothelial growth factor (VEGF) and TNF- $\alpha .^{12}$ Therefore, a new strategy constituting a more physiologically relevant way of improving the performance of engineered tissues may involve utilizing the power of macrophages. ${ }^{13}$ However, few studies have investigated how the interactions between biomaterials and immune cells affect the subsequent osteogenesis and angiogenesis of seeding cells.

Graphene is a two-dimensional (2D) carbonaceous material that has been widely applied in many research fields due to its unique physical, chemical, and mechanical properties. Graphene oxide (GO) is a product of the oxidation and exfoliation of graphite. In recent years, researchers have shown that GO can promote the osteogenic differentiation of bone marrow mesenchymal stem cells (BMSCs); therefore, it is recognized as a potential novel biomaterial for bone regeneration. ${ }^{14,15}$ GO has been found to elicit an immunomodulatory response that can affect the macrophage phenotype. ${ }^{16}$ However, the osteo-immunomodulatory effects of GO on the osteogenic differentiation of BMSCs and angiogenic differentiation of endothelial cells (ECs) are largely unknown.

In this study, we evaluated the influence of GO on the response of macrophages, osteogenic differentiation of BMSCs, and angiogenic differentiation of human umbilical vein endothelial cells (HUVECs). We also further investigated the possible mechanisms underlying the osteo- and angio-immunomodulatory effects of GO.

\section{Materials and methods \\ Fabrication of GO nanocomposites}

GO powder was purchased from Tanfeng Tech (Suzhou, China). It was synthesized using a modified Hummer's method. ${ }^{17}$ Briefly, $2 \mathrm{~g}(\mathrm{w} / \mathrm{v})$ graphite flakes and $2 \mathrm{~g}(\mathrm{w} / \mathrm{v}) \mathrm{NaNO}_{3}$ were added to $90 \mathrm{~mL}$ of concentrated $\mathrm{H}_{2} \mathrm{SO}_{4}(98 \%)$ and mixed under constant stirring for 4 hours at $4{ }^{\circ} \mathrm{C}$. Next, $12 \mathrm{~g} \mathrm{(w/v)}$ of potassium permanganate was added to the homogeneous solution under stirring conditions. Then, $184 \mathrm{~mL}$ of water was slowly added to this mixture and stirred for 2 hours at $4^{\circ} \mathrm{C}$. The mixture was further stirred at $35^{\circ} \mathrm{C}$ for an additional 2 hours, followed by refluxing at $98^{\circ} \mathrm{C}$ for 10 minutes. The temperature of the system was shifted to $25^{\circ} \mathrm{C}$ and maintained for an additional 2 hours. Then, $40 \mathrm{~mL}$ of hydrogen peroxide solution was added in drops to the concentrated mixture, which resulted in a color change to bright yellow. A total of $400 \mathrm{~mL}$ of water was added to the mixture and stirred for 5 hours. GO particles were obtained by centrifugation, and were then washed with $10 \% \mathrm{HCl}$ and deionized water several times. The collected product was freeze-dried overnight to obtain GO powder. The GO powder was dissolved in $0.1 \mathrm{~mol} / \mathrm{L}$ of hydrochloric acid solution.

\section{Physiochemical characterization of GO}

The morphology of the GO samples was visualized by atomic force microscopy (AFM) (MultiMode; Veeco, New York, NY, USA) and transmission electron microscopy (TEM) (JEM-2100F; JEOL, Tokyo, Japan). Fourier-transform infrared (FTIR) spectrum was applied to identify the presence of functional groups on the surface of GO samples. The spectrum was taken from 4,000 to $400 \mathrm{~cm}^{-1}$ on a LabRAM HR Evolution instrument (Horiba Jobin Yvon, Palaiseau, France). GO samples were also analyzed by confocal microRaman spectroscopy, as described previously.

\section{Viability of RAW 264.7 cells cultured in the presence of GO}

The murine-derived macrophage cell line RAW 264.7 cells were purchase from American Type Culture Collection (ATCC). RAW 264.7 cells were cultured in 96-well plates $\left(10^{3}\right.$ cells/well $)$ and treated with $0.1,1$, and $10 \mu \mathrm{g} / \mathrm{mL}$ GO in $\alpha$-minimum essential medium ( $\alpha$-MEM) for 1 and 2 days. Cell proliferation was measured using a cell counting kit- 8 assay (CCK-8; Dojindo, Kumamoto, Japan) and MTT (3-(4,5-dimethyl)thiazol-2-yl-2,5-dimethyltetrazolium bromide) following the manufacturer's instructions. For CCK-8 assay, the medium was replaced with $100 \mu \mathrm{L}$ of $\alpha$-MEM and $10 \mu \mathrm{L}$ of CCK-8 at each time point. After a 4-hour incubation at $37^{\circ} \mathrm{C}$, the absorbance at $450 \mathrm{~nm}$ was determined using a model 680 microplate reader (Bio-Rad Laboratories Inc., Hercules, CA, USA). As for MTT, the medium was replaced with $200 \mu \mathrm{L}$ MTT solution $(5 \mathrm{mg} / \mathrm{mL})$ at each time point. The cells were cultured for another 5 hours. During this period, viable cells could reduce the MTT to formazan pigment, which was dissolved by $800 \mu \mathrm{L}$ dimethyl sulfoxide (DMSO) after removal of the culture medium. The absorbance at $490 \mathrm{~nm}$ was recorded using a model 680 microplate reader (Bio-Rad Laboratories Inc.).

\section{Intracellular localization of GO in RAW 264.7 cells}

RAW 264.7 cells were seeded in a 6-well plate at a density of $10^{5}$ cells/well. After 24 hours of incubation, the culture 
medium was replaced with $2 \mathrm{~mL}$ of $\alpha$-MEM (with $10 \%$ FBS) containing $1 \mu \mathrm{g} / \mathrm{mL}$ GO. After a 24-hour incubation period, the media was removed and the cells were collected into a 1.5-mL EP tube. The collected cells were washed three times with PBS and then pre-fixed with $4 \%$ glutaraldehyde at $4{ }^{\circ} \mathrm{C}$ overnight. After washing with $0.1 \mathrm{M}$ PBS, $1 \%$ osmium tetraoxide was applied for 1 hour to postfix the cells. The cells were then stained with $1 \%$ uranyl acetate for 1 hour and dehydrated with a series of ethanol concentrations. Then, the cells were treated with a series of Spurr's resin concentrations, infiltrated in Spurr's resin at $70^{\circ} \mathrm{C}$ for 24 hours, and sectioned with an ultramicrotome. The sections were observed by TEM (HT7700; Hitachi, Tokyo, Japan).

\section{Inflammatory response of macrophages cultured in the presence of GO}

Macrophage RAW 264.7 cells were seeded in a 6-well plate at a density of $1.5 \times 10^{5}$ cells/well. After a 24 -hour incubation, the culture medium was replaced with $1.5 \mathrm{~mL}$ of $\alpha$-MEM (with $10 \%$ FBS) containing $1 \mu \mathrm{g} / \mathrm{mL} \mathrm{GO}$. After incubating for another day, the supernatant was collected by centrifugation at 3,000 rpm for 10 minutes. The collected supernatant was used as conditioned medium for growing BMSCs and HUVECs. Total RNA was isolated using an RNAiso reagent (TaKaRa Bio Inc., Shiga, Japan). Reverse transcription was performed using $2 \mu \mathrm{g}$ of total RNA, Prime ScriptRT kit reagents, and gDNA Eraser (TaKaRa Bio Inc.). The levels of mRNAs encoding inflammatory cytokines (IL-6, IL-1 $\beta$, interferon [INF]- $\gamma$, oncostatin M [OSM], TNF- $\alpha$, inducible nitric oxide synthase [iNOS], IL-10, and arginase), Toll-like receptor (TLR) signaling pathway proteins (MyD88, TICAM-1, TICAM-2, and IKB), osteogenic cytokines (bone morphogenetic protein-2 [BMP2], OSM, and TGF $\beta$ ), and angiogenic cytokines (VEGF) were determined using a StepOnePlus real-time PCR system (Thermo Fisher Scientific, Waltham, MA, USA) and SYBR Premix Ex Taq (TaKaRa Bio Inc.) under the following conditions: $95^{\circ} \mathrm{C}$ for 30 seconds followed by 40 cycles of $95^{\circ} \mathrm{C}$ for 5 seconds and $60^{\circ} \mathrm{C}$ for 30 seconds. Glyceraldehyde 3-phosphate dehydrogenase (GAPDH) served as an internal control and allowed for differences among samples to be adjusted. DNA concentrations were calculated using the $2^{-\Delta \Delta \mathrm{Ct}}$ method. All primers used in this experiment were synthesized by Sangon Biotech (Shanghai, China) and are listed in Table 1.

Table I Primer sequences for real-time quantitative PCR

\begin{tabular}{|c|c|c|c|c|}
\hline Genes & $\begin{array}{l}\text { Gene bank } \\
\text { number }\end{array}$ & Forward primer & Reverse primer & $\begin{array}{l}\text { Product } \\
\text { size }\end{array}$ \\
\hline GAPDH & DQ403057.I & CTCAGTTGCTGAGGAGTCCC & ATTCGAGAGAAGGGAGGGCT & 60 \\
\hline IL-6 & $\mathrm{BCI} 38766.1$ & GACAAAGCCAGAGTCCTTCAGA & TGTGACTCCAGCTTATCTCTTGG & 99 \\
\hline IL-I $\beta$ & NM_00836I.4 & GCCACCTTTTGACAGTGATGAG & GACAGCCCAGGTCAAAGGTT & 95 \\
\hline INF- $\gamma$ & NM_008337.4 & CAGCAACAGCAAGGCGAAAA & TCATTGAATGCTTGGCGCTG & 90 \\
\hline TNF- $\alpha$ & AY423855.I & GATCGGTCCCCAAAGGGATG & ACTGATGAGAGGGAGGCCAT & 86 \\
\hline iNOS & U43428.I & GAGCCACAGTCCTCTTTGCT & CAACCTTGGTGTTGAAGGCG & 111 \\
\hline IL-10 & NM_010548.2 & GGCCCAGAAATCAAGGAGCA & AATCGATGACAGCGCCTCAG & 62 \\
\hline Arginase & U5I805.I & CTGCGACCCAAGAAGACTAGA & TTGAGAAAGGCGCTCCGATAA & 164 \\
\hline RUNX2 & NM_00I024630.3 & CAAGTGGCCAGGTTCAACGA & TGTGAAGACCGTTATGGTCAAAGTG & 77 \\
\hline COLIAI & NM_000088.3 & СCTGCTGGCAAGAGTGGTGAT & CAAGTTCCGGTGTGACTCGTG & 145 \\
\hline OCN & NM_199173.5 & AGGACCCTCTCTCTGCTCAC & GCTCACACACСТСССТ & 68 \\
\hline OPN & J04765.I & GACGAGCACATCACCTCACA & GGCTTCAGCACTCTGGTCAT & 58 \\
\hline Osterix & AF47798I.I & GCTCCTTGGGACCCGTTC & GAGTTGTTGAGTCCCGCAGA & 112 \\
\hline MyD88 & U7045I.I & CATACCCTTGGTCGCGCTTA & TCCGAGGGTTCAAGAACAGC & 115 \\
\hline TICAMI & NM_I82919.3 & СТTCСССACAGTCCCAATCC & GAACCATCTGGGCATGGTGA & 66 \\
\hline TICAM2 & NM_021649.7 & TTGCTCAGTGCGAGAGGAAG & GTGGTACTGCTTGACCACGA & 165 \\
\hline TGF $\beta I$ & NM_00I036.4 & GTCACTGGAGTTGTACGGCA & AGCCCTGTATTCCGTCTCCT & 73 \\
\hline BMP2 & NM_00I200.3 & TGCTTCTTAGACGGACTGCG & CACTAGAAGACAGCGGGTCC & 66 \\
\hline OSM & NM_020530.5 & TCATCCTGAGCATGGCACTG & CGTGAGGTTCGCCTGATTCT & 63 \\
\hline VEGF & AY04758I.I & AACGATGAAGCCCTGGAGTG & GCTGGCTTTGGTGAGGTTTG & 117 \\
\hline CD3। & NM_000442.4 & GACTGAACCTGTCCTGCTCC & GGATGGTGAAGTTGGCTGGA & 70 \\
\hline MMP9 & NM_004994.2 & AAATCCCCACTGGGACCAAC & TTGTATCCGGCAAACTGGCT & 92 \\
\hline VEGFRI & EU826562.1 & TGCCTGTGGAAGAAATGGCA & GCTGTAGAAGCCAGTGTGGT & 92 \\
\hline VEGFR2 & EU826563.I & CGGAAATGACACTGGAGCCT & AATGACCGAGGCCAAGTCAG & 61 \\
\hline PDGFR $\alpha$ & NM_006206.5 & TGCGTAAGAGCAAAAAGCGA & GCTCCGAATCTCCCAGTGTC & 57 \\
\hline PDGFR $\beta$ & NM_002609.3 & GGATCGCTCTGTGAGCAACT & GGCTCTCTCСTCСTCСTTGT & 102 \\
\hline
\end{tabular}


Osteogenic gene/protein expression and mineralization of BMSCs by the stimulation of GO/RAW 264.7 cells grown in conditioned culture medium

The BMSCs used in our study were obtained from patients with femoral fracture. All donors were given written informed consent prior to the collection of their bone marrow and the bone marrow was collected during the process of femoral fracture fixation using reamed intramedullary nailing. All protocols were approved by the Ethics board of the Second Affiliated Hospital, Zhejiang University School of Medicine. To investigate the effect of GO-stimulated macrophages on the osteogenic differentiation of BMSCs, we collected the culture supernatant from RAW 264.7 cells to use as conditioned medium for further culture. BMSCs were seeded into 6 -well plates $\left(4 \times 10^{4}\right.$ cells/well) containing osteogenic conditioned induction medium (OIM: RAW 264.7 cells conditioned medium [with or without $1 \mu \mathrm{g} / \mathrm{mL}$ GO-stimulated], $100 \mathrm{nM}$ dexamethasone, $0.2 \mathrm{mM}$ ascorbic acid, and $10 \mathrm{mM} \beta$-glycerophosphate). Total RNA was isolated from cells cultured for 3 days using the RNAiso reagent (TaKaRa Bio Inc.). Reverse transcription was performed using $2 \mu \mathrm{g}$ of total RNA, Prime ScriptRT kit reagents, and gDNA Eraser (TaKaRa Bio Inc.). The levels of mRNAs encoding ALP, osteocalcin (OCN), runt-related transcription factor 2 (RUNX2), osteopontin (OPN), osterix, and collagen $\alpha 1$ type I (COL1A1) were determined using a StepOnePlus real-time PCR system (Applied Biosystems Inc.) and the SYBR Premix Ex Taq (TaKaRa Bio Inc.) under the following conditions: $95^{\circ} \mathrm{C}$ for 30 seconds followed by 40 cycles of $95^{\circ} \mathrm{C}$ for 5 seconds and $60^{\circ} \mathrm{C}$ for 30 seconds. GAPDH served as an internal control and allowed for differences among samples to be adjusted. DNA concentrations were calculated using the $2^{-\Delta \Delta C t}$ method (Livak and Schmittgen, 2001). ${ }^{36}$ All primers used in this experiment were synthesized by Sangon Biotech and are listed in Table 1.

Cells were lysed in RIPA lysis buffer (Beyotime, Shanghai, China) and subjected to SDS-PAGE on $10 \%$ polyacrylamide gels. The proteins were blotted onto poly (vinylidene fluoride) (PVDF) membranes (Millipore, Billerica, MA, USA). After blocking in 5\% non-fat milk for 2 hours, the membranes were incubated overnight at $4{ }^{\circ} \mathrm{C}$ with antibodies specific for GAPDH $(1: 1,500$; Cell Signaling Technology, Danvers, MA, USA), RUNX2 (1:1,600; Cell Signaling Technology), or COL1A1 (1:5,000; Abcam, Cambridge, UK), and then incubated with horseradish-peroxidase-conjugated goat anti-rabbit $\operatorname{IgG}(1: 5,000$; Beyotime) (the secondary antibody) for 2 hours at room temperature. Immunoreactive bands were detected using an enhanced chemiluminescence detection reagent (Millipore) and further visualized by exposing the blots to X-ray film (Bio-Rad Laboratories Inc.) for 0.1-2 minute. Protein expression was quantified by measuring the ratio of the absorbance of the protein of interest to that of the internal control (GAPDH).

To investigate mineral deposition, Alizarin red S (ARS) (Cyagen Biosciences, Guangzhou, China) was used. BMSCs were seeded in 12-well plates in GO/RAW 264.7-conditioned osteogenic medium. After 10 days of culture, BMSCs were fixed in 4\% paraformaldehyde (Sangon Biotech) for 10 minutes at room temperature, washed with distilled water, treated with ARS $(0.5 \%)$ for 30 minutes at room temperature, and rinsed with distilled water. The absorbances at $560 \mathrm{~nm}$ of 200- $\mu \mathrm{L}$ suspensions of stained cells in 96-well plates were measured using a microplate reader (ELX808; BioTek, Winooski, VT, USA). The readings were normalized to the total protein concentrations.

To investigate mineral deposition, the ALP stain (Beyotime) was used. After 3 days of culture, cells were fixed with $4 \%$ paraformaldehyde, washed twice with PBS, and stained using the BCIP/NBT Alkaline Phosphatase Color Development Kit (Beyotime). For measurement of ALP activity, cells were lysed with lysis buffer consisting of $20 \mathrm{mM}$ Tris- $\mathrm{HCl}$ (pH 7.5), $150 \mathrm{mM} \mathrm{NaCl}$, and 1\% Triton X-100. ALP activity was determined using the ALP Activity Assay (Beyotime) according to the manufacturer's instructions.

\section{Signal pathways of BMSCs involved in the immunomodulatory properties of GO}

To investigate the possible mechanisms underlying the immunomodulatory properties of GO in the osteogenesis of BMSCs, the expression levels of COL1A1, $\mathrm{p}-\beta$-Catenin, nuclear factor- $\mathrm{\kappa B}(\mathrm{NF}-\kappa \mathrm{B}), \mathrm{p}-\mathrm{Smad} 1 / 5 / 9$, and VEGF were analyzed by Western blot. As described in the previous section, BMSCs were treated with OIM (10\% FBS, $100 \mathrm{nM}$ dexamethasone, $0.2 \mathrm{mM}$ ascorbic acid, and $10 \mathrm{mM}$ $\beta$-glycerophosphate) for 3 days.

\section{Angiogenesis of HUVECs by stimulation with GO/RAW 264.7-conditioned culture medium}

To investigate the effect of GO-stimulated macrophages on the angiogenic differentiation of HUVECs, we collected the culture supernatant from the growth of RAW 264.7 cells as conditioned medium for further culture. HUVECs were 
purchased from ScienCell Research Laboratories (Carlsbad, CA, USA). HUVECs were seeded into 6-well plates ( $10^{5}$ cells/well) and cultured with GO-conditioned medium for 3 days. Then, angiogenic genes including CD31, MMP9, and VEGF pathway-related factor genes (VEGFR1, VEGF2, PDGFR $\alpha$, and PDGFR $\beta$ ) were analyzed by RT-PCR.

To investigate the angiogenic effects of GO-conditioned medium on HUVECs, we performed a tube formation assay. Briefly, $10^{5}$ HUVECs were seeded into 24-well plates precoated with Matrigel (Geltrex; Thermo Fisher Scientific, Waltham, MA, USA) and incubated with GO/RAW 264.7conditioned medium or normal culture medium. Images were taken after 8 hours of incubation. The number of bifurcations per field was quantified by ImageJ software.

\section{Statistical analysis}

Statistical analysis was performed using SPSS software (ver. 17.0; SPSS Inc., Chicago, IL, USA). Statistical significance was evaluated using the non-parametric Mann-Whitney and Wilcoxon signed-rank tests. All data are presented as means $\pm \mathrm{SD}$. A $P$-value $\leq 0.05$ was considered to indicate statistical significance.

\section{Results}

\section{Fabrication and characterization of GO}

GO nanocomposites were characterized by AFM, X-ray diffraction (XRD), TEM, and Raman spectroscopy (Figure 1). The morphology of GO nanosheets following ultrasonic treatment was observed by AFM and TEM (Figure 1). The GO nanosheets had 2D irregularly shaped flakes with diameters under $500 \mathrm{~nm}$. The XRD spectra of GO showed a sharp peak at $2 \theta=10.84^{\circ}$, which was due to the oxidation of graphite (Figure 1). The Raman spectra of GO also showed two intense and prominent peaks, indicating the $\mathrm{G}$ band and $\mathrm{D}$ band (Figure 1). These two bands arose from the $\mathrm{sp}^{2}$ carbon vibrations at 1,580 and $1,345 \mathrm{~cm}^{-1}$. The $\mathrm{D}$ and $\mathrm{G}$ band peaks represented the defects in the GO sample and were characteristic of the breathing mode of aromatic rings.

\section{Viability and internalization of RAW 264.7 cells cultured with GO}

To evaluate the immune modulatory properties of GO, RAW 264.7 cells were chosen for in vitro study. A CCK-8 assay was performed to study the effects of GO on the viability of RAW cells at days 1 and 2. Our results showed that GO had
A

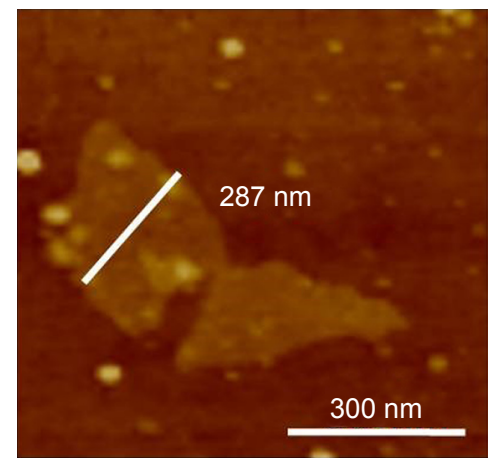

C

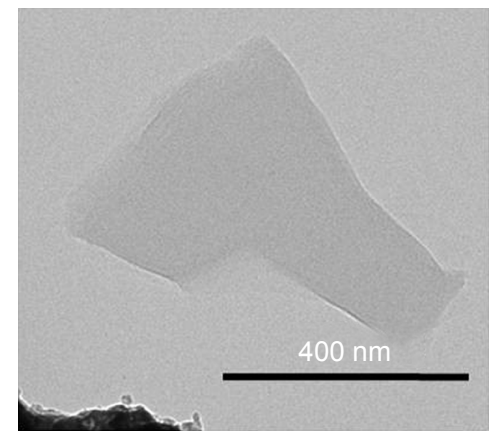

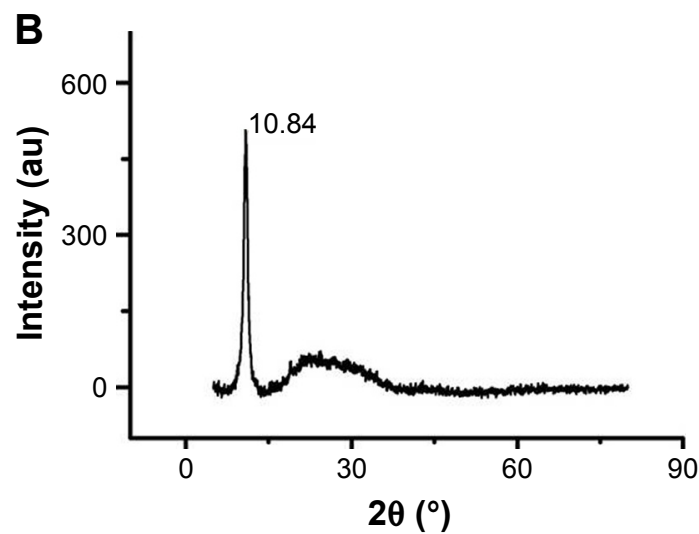

D

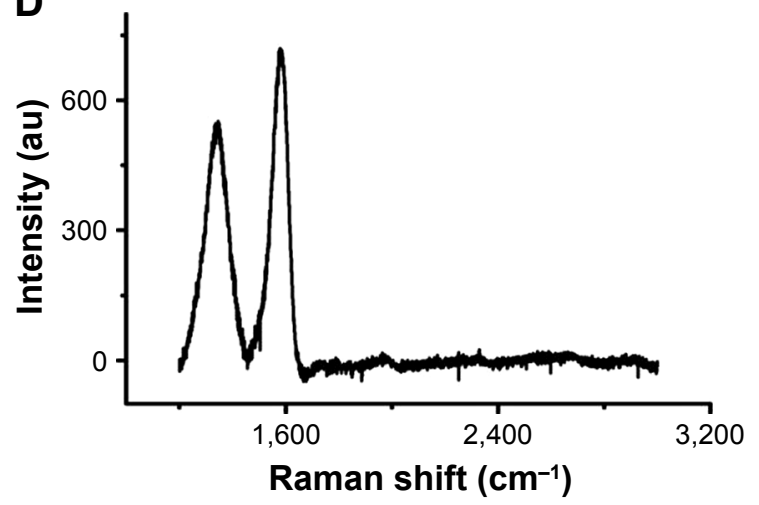

Figure I (A) Representative two-dimensional AFM image of GO; (B) XRD pattern of GO; (C) TEM image of GO; and (D) Raman spectra of GO. Abbreviations: GO, graphene oxide; AFM, atomic force microscopy; XRD, X-ray diffraction; TEM, transmission electron microscopy. 
no toxicity to macrophages. Furthermore, low concentrations of GO could stimulate macrophage proliferation (Figure 2). The MTT showed similar results (Figure S1).

To understand the mechanisms underlying the immunomodulatory properties of GO, GO intracellular localization was assessed by TEM. TEM revealed a large uptake of GO by the cells when cultured with $1 \mu \mathrm{g} / \mathrm{mL}$ GO (Figure 3). Figure $3 \mathrm{~A}$ shows that the $\mathrm{GO}$ sheets were located inside cells. Figure $3 \mathrm{~B}$ shows that the GO sheets were phagocytosed by phagosomes within the cytosol.

\section{Inflammatory gene expression of RAW cells cultured with GO}

To investigate the immune modulatory properties of GO, RAW 264.7 cells were cultured with or without GO. The gene expression results showed that the pro-inflammatory cytokines IL-6, IL-1 $\beta$, INF- $\gamma$, OSM, and TNF- $\alpha$ were increased. However, the level of the anti-inflammatory cytokine IL-10 did not change significantly (Figure 4).

\section{GO/RAW 264.7-conditioned culture medium promotes osteogenic \\ differentiation of BMSCs}

The expression of osteogenesis-related genes in GO/RAW 264.7-conditioned culture medium was assessed by RT-PCR. The results showed that the Runx2, Colla1, Ocn, Opn, and Osterix genes were significantly upregulated by conditioned medium from RAW 264.7 cells exposed to GO compared to the control groups (Figure 5A). The Western blot showed that osteogenic-related proteins, including Runx2 and Col1A1, were significantly upregulated (Figure 5B and C). ALP activity and staining revealed higher ALP activity in BMSCs treated

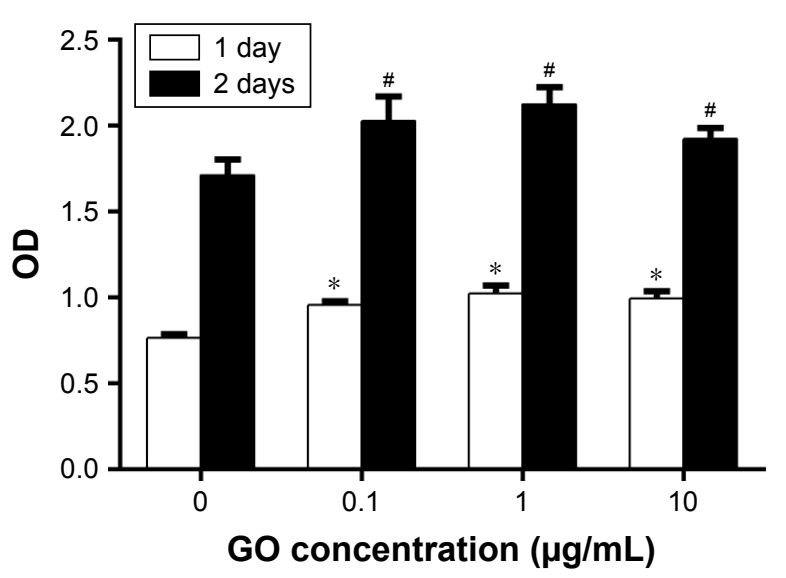

Figure 2 The effect of GO on the proliferation of RAW 264.7 cells $(* P<0.05$ versus $0 \mu \mathrm{g} / \mathrm{mL}$, day I and $\# P<0.05$ versus $0 \mu \mathrm{g} / \mathrm{mL}$, day 2).

Abbreviation: GO, graphene oxide.
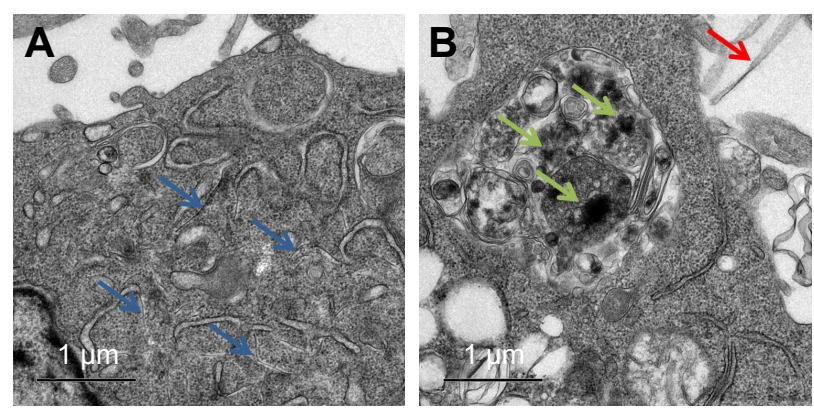

Figure 3 TEM images of GO sheets internalized by macrophages. (A) Blue arrows indicate GO sheets within the cytosol. (B) Green arrows indicate GO sheets phagocytosed by phagosomes; and the red arrow indicates $\mathrm{GO}$ sheets around the cells. Abbreviations: GO, graphene oxide; TEM, transmission electron microscopy.

with conditioned medium from RAW 264.7 cells exposed to GO relative to the control group (Figure 5D and E). ARS staining, which is used to determine the amount of calcium deposits, showed similar results (Figure 5F and G).

\section{GO/RAW 264.7-conditioned culture medium promotes osteogenic differentiation of BMSCs through signaling pathways}

To explore the possible mechanisms of how GO/RAW 264.7 conditioned culture medium promote the osteogenesis of MSCs, we performed RT-PCR and Western blot analysis on RAW 264.7 cells cultured with or without GO. The results showed that the expression levels of MyD 88, BMP2, VEGF, and OSM were significantly upregulated in RAW 264.7 cells stimulated with GO. The Western blot results showed that the expression levels of p-STAT3, p- $\beta$-Catenin, p-NF- $\kappa$ B,

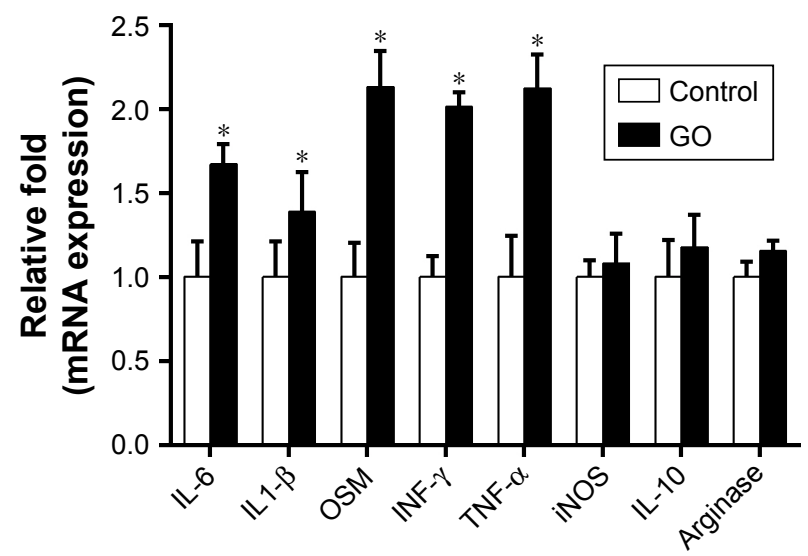

Figure 4 Inflammatory response of macrophages cultured with GO conditioned medium. RT-PCR showed that gene expression was increased. $* P<0.05$ versus the control group.

Abbreviations: GO, graphene oxide; IL, interleukin; INF- $\gamma$, interferon gamma; iNOS, inducible nitric oxide synthase; OSM, oncostatin M; TNF- $\alpha$, tumor necrosis factor alpha; RT-PCR, reverse transcriptase-polymer chain reaction. 
A

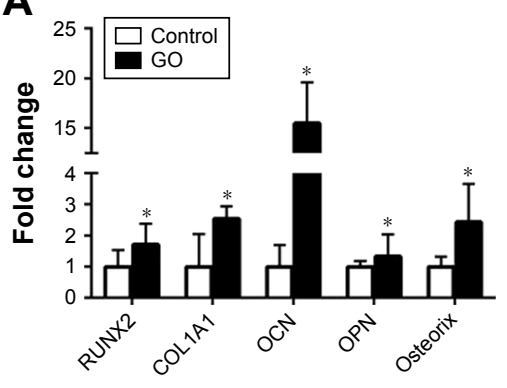

D

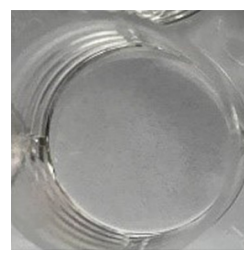

Control

F

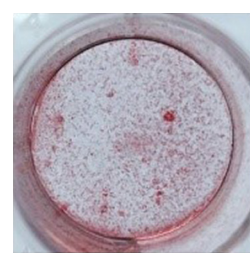

Control

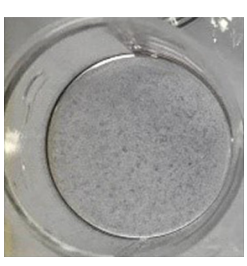

GO

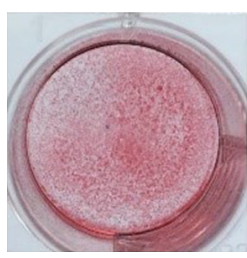

GO
B

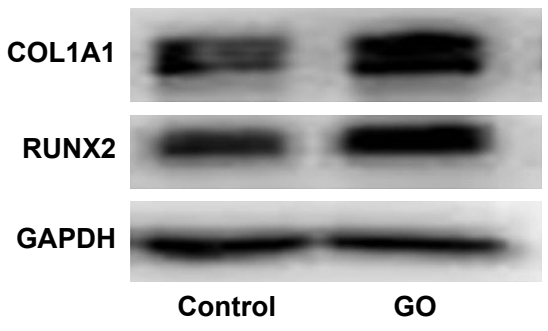

Control
C

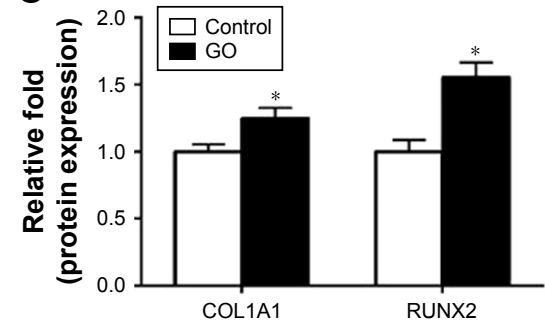

Figure 5 Osteogenic differentiation of BMSCs by stimulation with GO/RAW 264.7 conditioned culture medium.

Notes: (A) The expression levels of osteogenic mRNAs were determined by qPCR under conditions of osteogenic differentiation. (B and C) Western blotting analysis after osteogenic differentiation was used to determine the expression of Runx2 and CollAI proteins. (D) ALP in BMSCs was stained after osteogenic differentiation for 3 days. (E) ALP activity of BMSCs. (F) ARS staining after osteogenic differentiation for 9 days. (G) Mineralization was quantified by extracting ARS-stained cells. All data were confirmed by three repeated tests. $* P<0.05$ versus the control group.

Abbreviations: ALP, alkaline phosphatase; ARS, Alizarin red S; BMSCs, bone marrow stem cells; COLIAI, collagen $\alpha$ I type I; GAPDH, glyceraldehyde 3-phosphate dehydrogenase; GO, graphene oxide; OCN, osteocalcin; OPN, osteopontin; qPCR, quantitative PCR; RUNX2, runt-related transcription factor 2.

Smad1/5/9, and VEGF were significantly upregulated in BMSCs by GO-conditioned medium (Figure 6).

\section{The angiogenic effect of GO via immune modulation}

To investigate the angiogenic effect of GO via immune modulation, the expression of VEGF from RAW 264.7 cells was assessed. Our results showed that GO enhanced VEGF expression from RAW 264.7 cells. The ELISA results further confirmed the levels of VEGF in conditioned medium raised with the GO concentrations (Figure 7A). Then, we applied the GO/RAW 264.7-conditioned medium for HUVEC culture. The expression levels of angiogenic factors, including CD31 and MMP9, were upregulated by GO/RAW 264.7-conditioned medium stimulation. The expression levels of VEGF pathway-related factors, including VEGFR1, VEGF2, PDGFR $\alpha$, and PDGFR $\beta$, were increased (Figure 7B).
The endothelial tube formation assay is a classic in vitro angiogenesis test. Our results showed that HUVECs cultured in GO-conditioned medium had significantly enhanced tube formation after 8 hours of culture compared to the control group (Figure 7C-E).

\section{Discussion}

In this study, we found that low concentrations of GO stimulated the proliferation of RAW 264.7 cells. RAW 264.7 cells internalized GO by phagocytosis and were stimulated to undergo a M1 phenotype switch. The RAW 264.7 cells initiated the expression of several pro-inflammatory cytokines and created a favorable immune environment for osteogenesis and angiogenesis. The immune microenvironment induced by GO could stimulate osteogenic differentiation of BMSCs through OSM and NF- $\mathrm{KB}$ pathways. Furthermore, it stimulated the angiogenesis of HUVECs through the VEGF pathway, which is also important for bone regeneration. 


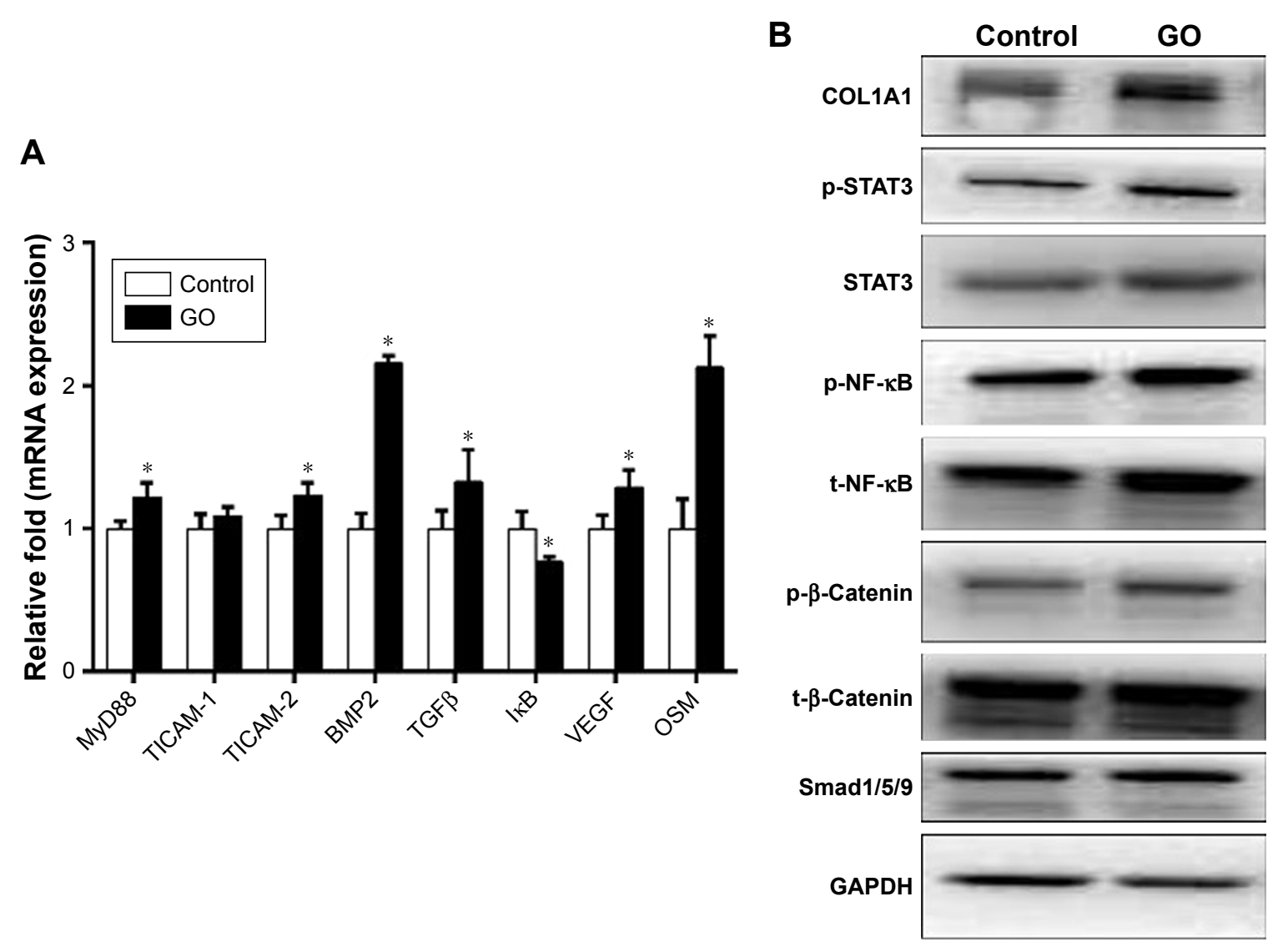

C

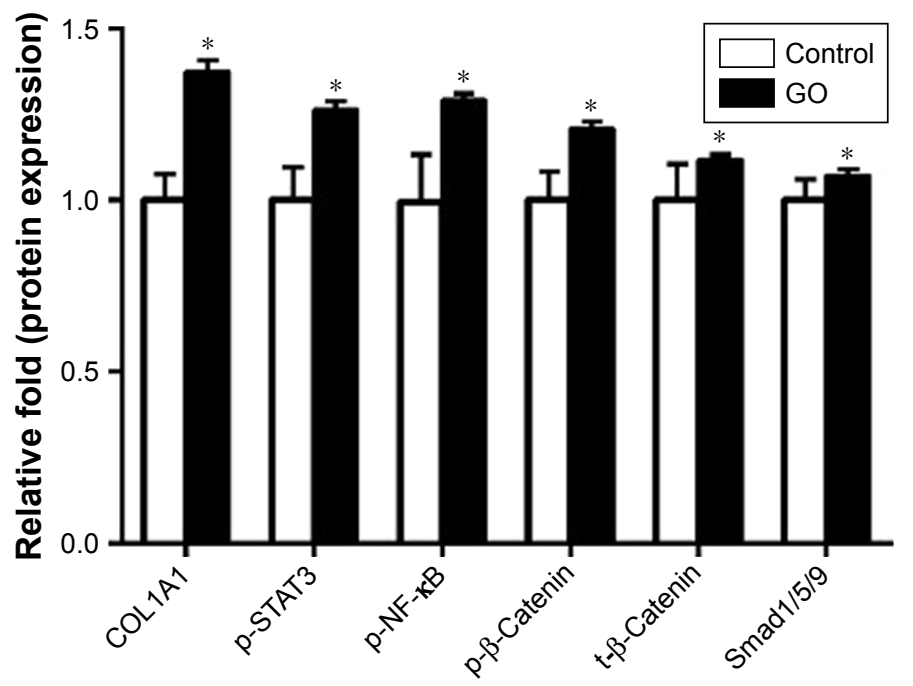

Figure 6 (A) Relative mRNA expression levels of MyD88, TICAM-I, TICAM-2, BMP2, TGF 3 , IאB, VEGF, and OSM from RAW 264.7 cells cultured in the presence of GO. (B, C) Western blot analysis of COLIAI, p- $\beta$-Catenin, p-NF-KB and SmadI/5/9 expression by BMSCs cultured in osteogenic medium and GO/RAW 264.7 conditioned osteogenic culture medium. $* P<0.05$ versus the control group.

Abbreviations: BMSCs, bone marrow stem cells; BMP2, bone morphogenetic protein-2; OSM, oncostatin M; TGF $\beta$, transforming growth factor $\beta$, VEGF, vascular endothelial growth factor.

The possible mechanisms of osteoimmunomodulatory effects of the GO-mediating osteogenesis and angiogenesis are summarized in Figure 8.

Macrophages are the first-line defense against foreign bodies and play a central role in the innate immune response.
Several studies have shown that biomaterials, including $\beta$-tricalcium phosphate, silica, and GO, can stimulate the immune response of macrophages after implantation. ${ }^{18-20}$ In our study, the GO sheets distributed around the RAW 264.7 cells or were up-taken and internalized by RAW 264.7 cells 

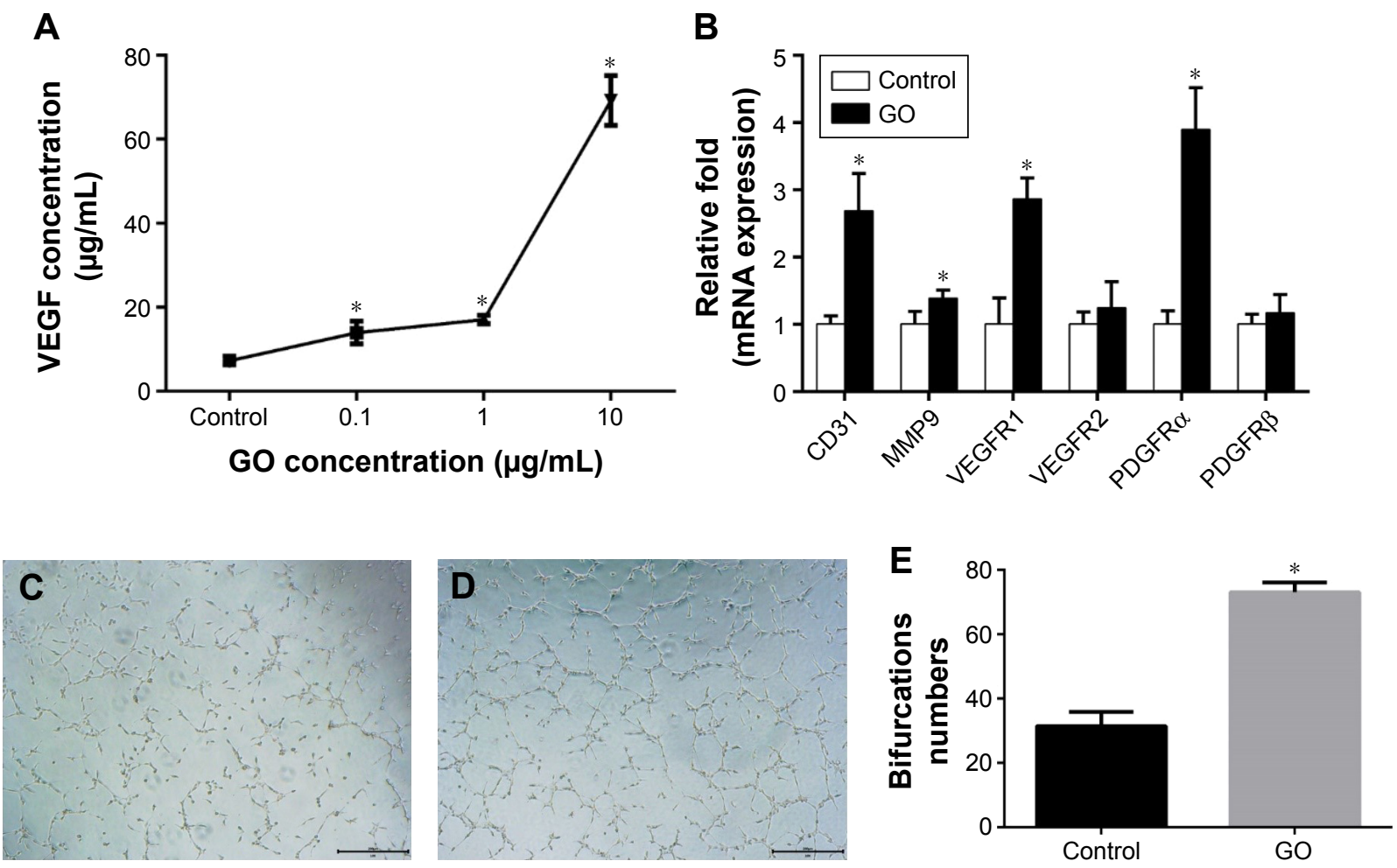

Figure 7 Angiogenic differentiation of HUVECs by stimulation with GO/RAW 264.7 conditioned medium.

Notes: (A) ELISA results showed that the levels of VEGF in condition medium raised with the GO concentrations. A tube formation assay was performed with normal culture medium. (B) RT-PCR showed that gene expression levels of angiogenic factors and VEGF pathway-related factors were increased compared to the control group $(* P<0.05)(\mathbf{C})$ and GO/RAW 264.7 (D) conditioned medium for 8 hours. $(\mathbf{E})$ Bifurcations numbers of the tubes.

Abbreviations: GO, graphene oxide; HUVECs, human umbilical vein endothelial cells; RT-PCR, reverse transcriptase-polymer chain reaction; VEGF, vascular endothelial growth factor.

in the cell matrix, as shown in the TEM image of Figure 3. The results in Figure 4 showed that GO stimulated RAW 264.7 cells to undergo an M1 phenotype switch, producing IL-6, IL-1 $\beta$, INF- $\gamma$, OSM, and TNF- $\alpha$. Our results showed that the surface makers $\mathrm{CD} 11 \mathrm{c}$ and $\mathrm{CC} 7$ of M1 were significantly upregulated in RAW 264.7 cells treated with GO. This was consistent with the literature. ${ }^{16}$ According to the results in Figure 6, MyD88 and TICAM-2 were significantly upregulated in the GO group. Therefore, we speculated that GO stimulated RAW 264.7 cells through the NF- $\mathrm{\kappa B}$ signal

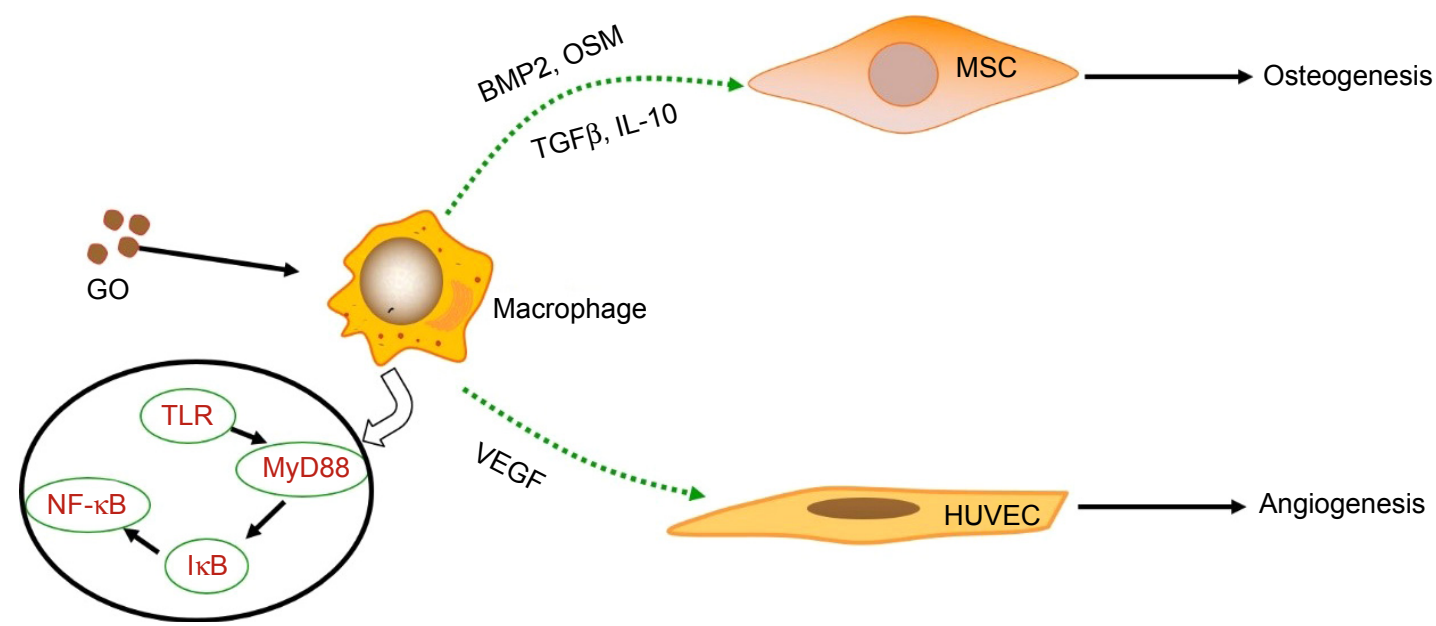

Figure 8 The possible mechanisms of osteoimmunomodulatory effects of the GO mediating osteogenesis and angiogenesis.

Abbreviations: BMP2, bone morphogenetic protein-2; GO, graphene oxide; HUVEC, human umbilical vein endothelial cell; IL, interleukin; MSC, mesenchymal stem cell; $\mathrm{NF}-\mathrm{KB}$, nuclear factor- $\mathrm{KB}$; OSM, oncostatin M; TGF $\beta$, transforming growth factor $\beta$; VEGF, vascular endothelial growth factor. 
pathway, which led to the activation of downstream cascades. Chen et $\mathrm{al}^{21}$ reported that the regulation of phagocytosis by TLRs was mediated by direct interaction with MyD88.

We found that the immune microenvironment modulated by GO could stimulate osteogenic differentiation of BMSCs. As shown in Figure 5, GO could not only stimulate early osteogenic gene expression, but also calcium deposition. This may be mediated by OSM and BMP2, which are secreted by RAW 264.7 cells. Generally, macrophages are divided into two phenotypes: classically activated inflammatory macrophages (M1) and alternatively activated inflammatory macrophages (M2). Traditionally, the M1 phenotype is known to elicit proinflammatory effects, which may cause bone resorption, while the M2 phenotype is associated with osteogenesis. ${ }^{22}$ However, in recent years, some studies have reported that the $\mathrm{M} 1$ phenotype is beneficial for osteogenesis through the activation of the OSM signaling pathway. ${ }^{23,24}$ Our results showed that the expression levels of OSM and BMP2 in RAW 264.7 cells in the GO group were significantly upregulated. The Western blot results showed that the expression levels of p-STAT3 and Smad1/5/9 were significantly upregulated. Several studies have reported that OSM can activate osteogenesis through activation of STAT3. ${ }^{25,26}$ In addition, BMP2 is a well-known growth factor that promotes osteogenesis, and has been used in clinical applications. ${ }^{27}$ BMP2 promotes osteogenic differentiation mainly through the Smad1/5/9 signal pathway. ${ }^{28}$ These results show that GO stimulates the formation of an immune microenvironment that is favorable for BMSC osteogenesis.

TNF- $\alpha$ has been shown to have dual effects on the osteogenic differentiation of BMSCs: it promotes osteogenic differentiation at low concentrations and inhibits osteogenic differentiation at high concentrations $(>10 \mathrm{ng} / \mathrm{mL}) .{ }^{29}$ Our results showed that GO stimulated RAW 264.7 cells to secrete very low concentrations $(<1 \mathrm{ng} / \mathrm{mL})$ of TNF- $\alpha$. Therefore, the range of concentrations of TNF- $\alpha$ in this immunomodulatory environment favors osteogenic differentiation of BMSCs.

Our results showed that the immune microenvironment modulated by GO could stimulate angiogenic differentiation of HUVECs. Angiogenesis is also important for bone tissue engineering. ${ }^{30,31}$ After tissue engineering, bone is implanted in vivo. A major challenge in the clinical evolution of bone substitutes is the maintenance of cell viability in the graft center, which mainly depends on the rapidity of host blood vessel invasion. ${ }^{30}$ Some researchers tried to combine osteogenic and angiogenic growth factors for bone regeneration; ${ }^{32,33}$ however, the high costs of the growth factors limited further clinical applications. In our study, we showed GO could not only promote osteogenesis of
BMSCs, but also stimulate angiogenesis of HUVECs. Our results indicate that GO-stimulated angiogenesis may activate downstream signaling pathways through VEGFR and PDGFR, resulting in the proliferation and angiogenesis of vascular ECs. A number of studies have shown that a combination of VEGFR and PDGFR with corresponding ligands can promote the proliferation and angiogenesis of vascular ECs by activating multiple signaling pathways, such as PI3K/ Akt, MAPK, and PLC- $\gamma \cdot{ }^{34,35}$ Therefore, GO can be used to stimulate the development of an immune microenvironment that is suitable for bone regeneration.

\section{Conclusion}

In conclusion, this study shows that the immune microenvironment induced by GO stimulates osteogenic differentiation of BMSCs through the OSM and NF- $\mathrm{BB}$ pathways, and angiogenesis of HUVECs through the VEGF pathway. Since both osteogenesis and angiogenesis are important for bone regeneration, GO may be used as an immunomodulatory agent for bone tissue engineering applications.

\section{Acknowledgments}

This study was supported by the Zhejiang Provincial Natural Science Foundation of China (grant nos LY18H060003, LY16H060003, and LQ18H050005), the National Nature Science Foundation of China (grant no 81871759, 81874007, 81802221, 81672147), Zhejiang medical and health science and technology plan project (grant nos 2015KYB182 and 2017KY382), and Hangzhou Science and Technology Committee Fund (grant no 20160533B61).

\section{Disclosure}

The authors report no conflicts of interest in this work.

\section{References}

1. Li JJ, Ebied M, Xu J, Zreiqat H. Current Approaches to Bone Tissue Engineering: The Interface between Biology and Engineering. $A d v$ Healthc Mater. 2018;7(6):e1701061.

2. Ge M, Ge K, Gao F, et al. Biomimetic mineralized strontium-doped hydroxyapatite on porous poly(l-lactic acid) scaffolds for bone defect repair. Int J Nanomedicine. 2018;13:1707-1721.

3. Alvim Valente C, Cesar Chagastelles P, Fontana Nicoletti N, et al. Design and optimization of biocompatible polycaprolactone/poly (1-lactic-co-glycolic acid) scaffolds with and without microgrooves for tissue engineering applications. J Biomed Mater Res A. 2018; 106(6):1522-1534.

4. Soancă A, Lupse M, Moldovan M, et al. Applications of inflammationderived gingival stem cells for testing the biocompatibility of dental restorative biomaterials. Ann Anat. 2018;218:28-39.

5. Haggerty AE, Maldonado-Lasunción I, Oudega M. Biomaterials for revascularization and immunomodulation after spinal cord injury. Biomed Mater. 2018;13(4):044105.

6. Hu Z, Ma C, Rong X, Zou S, Liu X. Immunomodulatory ECM-like Microspheres for Accelerated Bone Regeneration in Diabetes Mellitus. ACS Appl Mater Interfaces. 2018;10(3):2377-2390. 
7. Chen Z, Bachhuka A, Wei F, et al. Nanotopography-based strategy for the precise manipulation of osteoimmunomodulation in bone regeneration. Nanoscale. 2017;9(46):18129-18152.

8. Chen J, Li M, Yang C, et al. Macrophage phenotype switch by sequential action of immunomodulatory cytokines from hydrogel layers on titania nanotubes. Colloids Surf B Biointerfaces. 2018;163:336-345.

9. Spiller KL, Anfang RR, Spiller KJ, et al. The role of macrophage phenotype in vascularization of tissue engineering scaffolds. Biomaterials. 2014;35(15):4477-4488.

10. Roh JD, Sawh-Martinez R, Brennan MP, et al. Tissue-engineered vascular grafts transform into mature blood vessels via an inflammationmediated process of vascular remodeling. Proc Natl Acad Sci US A. 2010;107(10):4669-4674.

11. Brown BN, Badylak SF. Expanded applications, shifting paradigms and an improved understanding of host-biomaterial interactions. Acta Biomater. 2013;9(2):4948-4955.

12. Ribatti D, Crivellato E. Immune cells and angiogenesis. J Cell Mol Med. 2009;13(9A):2822-2833.

13. Niu Y, Li Q, Xie R, et al. Modulating the phenotype of host macrophages to enhance osteogenesis in MSC-laden hydrogels: Design of a glucomannan coating material. Biomaterials. 2017;139:39-55.

14. Jaidev LR, Kumar S, Chatterjee K. Multi-biofunctional polymer graphene composite for bone tissue regeneration that elutes copper ions to impart angiogenic, osteogenic and bactericidal properties. Colloids Surf B Biointerfaces. 2017;159:293-302.

15. Wang Q, Chu Y, He J, et al. A graded graphene oxide-hydroxyapatite/ silk fibroin biomimetic scaffold for bone tissue engineering. Mater $\mathrm{Sci}$ Eng C Mater Biol Appl. 2017;80:232-242.

16. Ma J, Liu R, Wang X, et al. Crucial Role of Lateral Size for Graphene Oxide in Activating Macrophages and Stimulating Proinflammatory Responses in Cells and Animals. ACS Nano. 2015; 9(10):10498-10515.

17. Hummers Jr WS, Offeman RE. Preparation of Graphitic Oxide. J Am Chem Soc. 1958;80(6):1339.

18. Shi M, Xia L, Chen Z, et al. Europium-doped mesoporous silica nanosphere as an immune-modulating osteogenesis/angiogenesis agent. Biomaterials. 2017;144:176-187.

19. Dudek I, Skoda M, Jarosz A, Szukiewicz D. The Molecular Influence of Graphene and Graphene Oxide on the Immune System Under In Vitro and In Vivo Conditions. Arch Immunol Ther Exp. 2016; 64(3):195-215.

20. Chen Z, Mao X, Tan L, et al. Osteoimmunomodulatory properties of magnesium scaffolds coated with $\beta$-tricalcium phosphate. Biomaterials. 2014;35(30):8553-8565.

21. Chen GY, Yang HJ, Lu CH, et al. Simultaneous induction of autophagy and toll-like receptor signaling pathways by graphene oxide. Biomaterials. 2012;33(27):6559-6569.
22. Pajarinen J, Lin T, Gibon E, et al. Mesenchymal stem cell-macrophage crosstalk and bone healing. Biomaterials. Epub 2018 Jan 2.

23. Shi M, Chen Z, Farnaghi S, et al. Copper-doped mesoporous silica nanospheres, a promising immunomodulatory agent for inducing osteogenesis. Acta Biomater. 2016;30:334-344.

24. Guihard P, Danger Y, Brounais B, et al. Induction of osteogenesis in mesenchymal stem cells by activated monocytes/macrophages depends on oncostatin M signaling. Stem Cells. 2012;30(4):762-772.

25. Feng X, Shen S, Cao P, et al. The role of oncostatin M regulates osteoblastic differentiation of dental pulp stem cells through STAT3 pathway. Cytotechnology. 2016;68(6):2699-2709.

26. Nicolaidou V, Wong MM, Redpath AN, et al. Monocytes induce STAT3 activation in human mesenchymal stem cells to promote osteoblast formation. PLoS One. 2012;7(7):e39871.

27. Rosen V. BMP2 signaling in bone development and repair. Cytokine Growth Factor Rev. 2009;20(5-6):475-480.

28. Wang CL, Xiao F, Wang CD, et al. Gremlin2 Suppression Increases the BMP-2-Induced Osteogenesis of Human Bone Marrow-Derived Mesenchymal Stem Cells Via the BMP-2/Smad/Runx2 Signaling Pathway. J Cell Biochem. 2017;118(2):286-297.

29. Wang L, Zhang J, Wang C, et al. Low concentrations of TNF- $\alpha$ promote osteogenic differentiation via activation of the ephrinB2-EphB4 signalling pathway. Cell Prolif. 2017;50(1):e12311.

30. Grosso A, Burger MG, Lunger A, Schaefer DJ, Banfi A, di Maggio N. It Takes Two to Tango: Coupling of Angiogenesis and Osteogenesis for Bone Regeneration. Front Bioeng Biotechnol. 2017;5:68.

31. Li J, Jahr H, Zheng W, Ren PG. Visualizing Angiogenesis by Multiphoton Microscopy In Vivo in Genetically Modified 3D-PLGA/ nHAp Scaffold for Calvarial Critical Bone Defect Repair. $J$ Vis Exp. 2017;127(127):e55381.

32. Sharmin F, Mcdermott C, Lieberman J, Sanjay A, Khan Y. Dual growth factor delivery from biofunctionalized allografts: Sequential VEGF and BMP-2 release to stimulate allograft remodeling. J Orthop Res. 2017;35(5):1086-1095.

33. Su YW, Chung R, Ruan CS, et al. Neurotrophin-3 Induces BMP-2 and VEGF Activities and Promotes the Bony Repair of Injured Growth Plate Cartilage and Bone in Rats. J Bone Miner Res. 2016;31(6): $1258-1274$.

34. Yuan X, Han L, Fu P, et al. Cinnamaldehyde accelerates wound healing by promoting angiogenesis via up-regulation of PI3K and MAPK signaling pathways. Lab Invest. 2018;98(6):783-798.

35. Shibuya M. VEGFR and type-V RTK activation and signaling. Cold Spring Harb Perspect Biol. 2013;5(10):a009092.

36. Livak KJ, Schmittgen TD. Analysis of relative gene expression data using real-time quantitative PCR and the 2(-Delta Delta C(T)) method. Methods 25. 2001:402-408 


\section{Supplementary material}

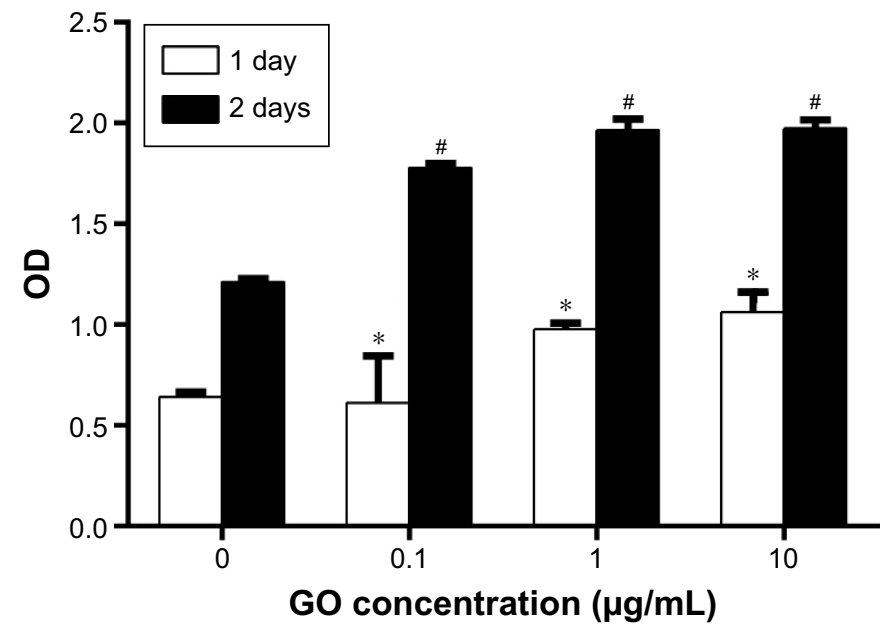

Figure SI The effect of $G O$ on the proliferation of RAW 264.7 cells $\left(* P<0.05\right.$ versus $0 \mu g / \mathrm{mL}$, day I and ${ }^{\# P}<0.05$ versus $0 \mu g / \mathrm{mL}$, day 2$)$. Abbreviation: GO, graphene oxide.

\section{Publish your work in this journal}

The International Journal of Nanomedicine is an international, peerreviewed journal focusing on the application of nanotechnology in diagnostics, therapeutics, and drug delivery systems throughout the biomedical field. This journal is indexed on PubMed Central, MedLine, CAS, SciSearch $\AA$, Current Contents ${ }^{\circledR} /$ Clinical Medicine,
Journal Citation Reports/Science Edition, EMBase, Scopus and the Elsevier Bibliographic databases. The manuscript management system is completely online and includes a very quick and fair peer-review system, which is all easy to use. Visit http://www.dovepress.com/ testimonials.php to read real quotes from published authors.

Submit your manuscript here: http://www.dovepress.com/international-journal-of-nanomedicine-journal 